\title{
What is the impact of the explicit knowledge of sequence regularities on both deterministic and probabilistic serial reaction time task performance?
}

\author{
Nicolas Stefaniak, Sylvie Willems, Stéphane Adam, And Thierry Meulemans \\ University of Liège, Liège, Belgium
}

\begin{abstract}
The aim of this study was to explore the role of prior explicit sequence knowledge by comparing its influence on serial reaction time (SRT) performance with either a deterministic or a probabilistic sequence. The results confirm that, with a deterministic sequence, preliminary explicit learning improves SRT performance. On the other hand, with a probabilistic sequence, the results show no advantage for SRT performance in explicitlearning conditions. In addition, by using the process dissociation procedure (Jacoby, 1991), we show that performance on a subsequent generation task was more sustained by controlled processes for participants in the explicit-learning conditions than for those in the incidental condition. On the whole, these results, showing that the influence of explicit knowledge can be suppressed in certain specific conditions, are consistent with the intervention of both implicit and explicit mechanisms in SRT tasks, and the results also show that their relative influence can be modulated by the particular demands of the task.
\end{abstract}

The question of the awareness associated with learning in so-called implicit-learning tasks is far from resolved. Simply put, there are two conflicting positions on this matter. According to some authors, the learning abilities revealed with implicit-learning paradigms reflect humans' ability to learn complex information without any consciousness of what they learn; in other words, such mechanisms reflect a kind of learning that occurs outside of conscious control and that is opposed by nature to explicit-learning mechanisms. Other authors have challenged this point of view - not necessarily the idea that some kinds of human learning may be implicit, but the validity of the evidence that has been presented to demonstrate the existence of such implicit-learning mechanisms (see Shanks, 2005, for a recent review).

The implicit-learning paradigm most often used in the context of the implicit/explicit debate has been the serial reaction time (SRT) task (Nissen \& Bullemer, 1987). In this task, participants are asked to respond as quickly as possible to stimuli appearing in different locations on a computer screen by pressing the corresponding keys of the keyboard; the participants are not told that the stream of stimuli corresponds to a repeating sequence. The idea that sequence learning in SRT tasks can be accounted for by the intervention of implicit-learning mechanisms has been supported by different types of evidence; amnesic patients' normal performance on such tasks has often been cited (e.g., Nissen, Willingham, \& Hartman, 1989;
Reber \& Squire, 1994, 1998; but see Curran, 1997b, and Wilkinson \& Shanks, 2004, for contradictory views). Other evidence comes from studies showing a dissociation between performance on the SRT task and performance on a subsequent explicit task (e.g., a recognition or generation task). If participants show sequence learning in the SRT task and no sequence knowledge in an explicit task, this result can be viewed as an argument in favor of the existence of sequence-learning mechanisms that occur outside of consciousness (e.g., Meulemans, Van der Linden, \& Perruchet, 1998; Perruchet, Bigand, \& BenoitGonin, 1997). However, these data must always be treated with caution. The problem here is the relative sensitivity of the implicit and explicit measures used; it could be hypothesized that this single dissociation simply reflects the fact that the explicit task is more difficult than the implicit one. It should be added that such a dissociation is not invariably observed in the SRT paradigm; depending on some characteristics of the material to be learned (e.g., its statistical structure, the length of the repeated sequence, the number of learning blocks, the response-stimulus interval [RSI], etc.), the development of explicit knowledge of the sequence may sometimes be the rule (Stadler \& Frensch, 1994). Moreover, several studies have shown a correlation between the degree of explicit knowledge and SRT performance (e.g., Hartman, Knopman, \& Nissen, 1989; Willingham, Nissen, \& Bullemer, 1989). Such observations are at the origin of the controversy about the 
involvement of explicit processes in the SRT paradigm and, more generally, in implicit-learning situations as a whole (Stadler \& Roediger, 1998; see also Willingham \& Goedert-Eschmann, 1999).

Destrebecqz and Cleeremans (2001) argued that if there is still no strong evidence of the existence of implicit learning, this is because the authors who have tried to evaluate the processes involved in the SRT task have tended to consider them as if they were pure processes: Performance on the SRT task would reflect the operation of implicit mechanisms and the existence of implicit knowledge, whereas performance on an explicit task (e.g., a recognition task) would reflect only the presence of explicit knowledge. However, many authors (see Jacoby, 1991) consider that there is virtually no cognitive task that is not sustained by both controlled/explicit and automatic/implicit processes. From that perspective, it is inappropriate to try to characterize a performance as being either implicit or explicit; on the contrary, the goal will be to separate out each kind of process's contribution to the performance. This is what the process dissociation procedure (PDP) proposed by Jacoby (1991) is meant to do.

Applied to a cued recall task, the basic principle of the PDP is to test the participant's knowledge in two conditions - the inclusion and the exclusion conditionsthat differ only according to the instructions given to the participant. In the inclusion condition, the participant has to complete the cue with the corresponding information that was previously encoded; if the participant cannot recall this information, then he/she must complete the cue with the first response that comes to mind. This condition is a facilitation condition in that the target response can be given by means of either the controlled (i.e., recollection) or the automatic (priming) processes. In the exclusion condition, the participant is instructed not to complete the cue with the target information that was previously learned, but to give a new response; if the participant cannot remember the target response, he/she gives the first response that comes to mind. So the exclusion condition is an interference condition, in which controlled and automatic processes act in opposition: In this situation, giving the target response (i.e., producing an error) indicates that the participant could not consciously remember the target, which was therefore given on the basis of automatic processes. ${ }^{1}$

Destrebecqz and Cleeremans (2001; see also Destrebecqz, 2004) applied the PDP to an SRT task in which they manipulated the RSI. In their study, after the SRT task, participants had to perform a free generation task. In the inclusion condition, they were asked to generate 96 items that resembled the training sequence as much as possible and to rely on their intuition when they felt unable to recollect the location of the next stimulus. In the exclusion condition, the participants had to generate 96 trials that avoided reproducing the sequential regularities. The authors showed that implicit processes played a greater role in the performance of the participants who responded to a sequence with no RSI. Again, these results were interpreted as strong evidence of the existence of implicit-learning mechanisms that differ from explicit ones. Note, however, that Wilkinson and Shanks (2004) were unable to replicate Destrebecqz and Cleeremans's (2001) results; in a no-RSI condition, they found that sequence knowledge could be accessible to consciousness.

Different kinds of data can be adduced concerning the influence of explicit mechanisms on SRT performance. The first type relates to the observation that, in SRT tasks, participants who can demonstrate after the task that they possess explicit knowledge of the sequence perform better on the SRT task (e.g., Cherry \& Stadler, 1995). This observation suggests at least that there is a relationship between implicit sequence learning and certain explicit mechanisms, even though it cannot be taken as strong proof of the influence of explicit knowledge on SRT performance (it could also be suggested that the probability of explicit knowledge's emerging depends on the quality and strength of the previous implicit sequence learning; see the results of Willingham, Greeley, \& Bardone, 1993).

More convincing are the studies showing that performance on the SRT task can be improved if the participants are given some preliminary explicit information. This preliminary information may take different forms: It may be general information about the presence of regularities in the stimulus presentation that may help the participant to respond more quickly to the stimuli (Curran, 1997a; Frensch \& Miner, 1994). In a few studies, the sequence itself has been presented to participants before the SRT task, and the participants have been given time to study the sequence before performing the so-called implicitlearning task (e.g., Cleeremans \& Jiménez, 1998; Curran \& Keele, 1993; Destrebecqz, 2004; Thomas \& Nelson, 2001). Most of these studies have shown that the difference in reaction time (RT) between the learned and the random sequences was greater for participants who had gained some explicit knowledge of the sequence than for those who had not. However, Jiménez, Méndez, and Cleeremans (1996) showed that this positive effect depends on the structural complexity of the sequence to be learned; by using a probabilistic sequence (determined on the basis of a finite-state grammar), they prevented participants who had previously been informed of the rule-governed nature of the stimuli and then were asked to try to discover these rules from improving their performance.

Other authors have given more detailed information to their participants. Curran and Keele (1993) let participants study a sequence of six elements for $1 \mathrm{~min}$ before the SRT task, which they performed under either a single-task or a dual-task condition. They showed that, under the singletask condition, participants who had the opportunity to learn the sequence before the SRT task improved their RTs in the task more than did those who experienced a classical incidental condition. Curran and Keele, who also found that this advantage disappeared when participants were placed in a dual-task condition, interpreted their results as favoring the existence of two independent learning mechanisms (see also Keele, Ivry, Mayr, Hazeltine, \& Heuer, 2003, for a more recent theoretical view of these two learning mechanisms; and Grafton, Hazeltine, \& Ivry, 
1995, who obtained neuroimaging data supporting this dual-system view).

Cleeremans and Jiménez (1998) adopted a methodology similar to that in Curran and Keele's (1993) study. They also let some of their participants study the sequence for 1 min before the SRT task. In addition, they manipulated different variables, including sequence type (deterministic vs. probabilistic). Cleeremans and Jiménez showed that preliminary explicit knowledge of the sequence helps only participants who have been trained with the deterministic sequence. They concluded that learning a probabilistic sequence depends essentially on implicit-learning mechanisms. Rauch et al. (1995) also showed in a PET study that the cerebral structures activated when the sequence is learned explicitly differ from those activated when the sequence is learned incidentally. According to this study, the regions involved in the incidental-learning condition are the right ventral premotor cortex, the right ventral caudate/nucleus accumbens, the right thalamus, and the bilateral Area 19; in the explicit-learning condition, the areas known to play a role in visual and language processes are involved (i.e., the primary visual and inferior parietal cortex).

Finally, Shea, Wulf, Whitacre, and Park (2001) obtained atypical results: They found that participants who were given such preliminary explicit instructions performed worse on the task than did participants in the classical incidental condition (see Howard \& Howard, 2001, for similar results). Shea et al. interpreted their result by arguing that explicit-learning mechanisms, because of their process limitations, may be negatively affected when task demands are too high in terms of processing resources; in such situations, implicit-learning mechanisms are more efficient. This interpretation has been challenged by Perruchet, Chambaron, and Ferrel-Chapus (2003), who argued that the explicit information Shea et al. gave to their participants before the task was not relevant; that is, it was information that the participants could not use to perform the task.

The main objective of this study was to further explore the impact of preliminary explicit knowledge on SRT performance, by manipulating both the kind of sequence (deterministic vs. probabilistic) and the kind of instructions given to participants. A secondary purpose was to determine, by using the PDP, whether participants' sequence knowledge assessed after the SRT task can be considered as implicit/automatic or explicit/controlled and to see whether this knowledge can be related to their performance during the SRT task.

With regard to the preliminary explicit knowledge, our aim was to induce participants to acquire a full explicit knowledge of the sequence before performing the SRT task, with both a deterministic (Experiment 1) and a probabilistic (Experiment 2) sequence. In both experiments, we trained our participants to learn the sequence explicitly until a learning criterion was reached (contrary to what had been done in previous studies, in which participants had to learn the sequence only for a certain period of time; see, e.g., Cleeremans \& Jiménez, 1998). To reach this goal with a probabilistic sequence (Experiment 2), we had to choose a probabilistic sequence whose features could be entirely learned explicitly. So we used a sequence adapted from Schvaneveldt and Gomez (1998), in which the irregular (i.e., low-probability) items were not selected randomly (unlike in Cleeremans \& Jiménez, 1998): The irregular element that might replace one element of the sequence (with a $20 \%$ probability) was always the same for that element. Thus, the participants could actually learn this information, and there was no element of the sequence that could not be predicted (taking into account the probabilistic nature of the sequence) on the basis of the knowledge that the participants had acquired beforehand about the structure of the sequence.

Our prediction was that, if the relevant information was given to the participants in advance, this explicit sequence knowledge would help them to respond more quickly in the deterministic SRT task; more specifically, we predicted that, with previous explicit knowledge of the sequence, the reaction time difference between the repeated and the random sequences should be more pronounced. We also predicted that this should not be the case with a probabilistic sequence, because, according to Schvaneveldt and Gomez (1998), in a probabilistic SRT task, "responses must be based on the imperative stimuli and not simply on knowledge of the sequence" (p. 177).

With regard to the PDP, an obvious prediction for the participants who learned the sequence explicitly before the SRT task was that they would show high levels of explicit knowledge in the post-SRT generation task. Nevertheless, for the participants who performed the SRT task with the probabilistic sequence, one possibility was that, in the generation task, such high levels of explicit knowledge would not be observed; this might happen if they could not use their preliminary explicit knowledge of the sequence during the SRT task and, so, this knowledge was not reinforced (or even maintained) during the task. We also predicted that, in the probabilistic condition, the explicit knowledge of the sequence demonstrated with the PDP would not be correlated with performance on the SRT task.

\section{EXPERIMENT 1}

In Experiment 1, we compared RTs in response to a deterministic sequence for two groups of participants: (1) a group who learned the sequence before the SRT task (explicit-learning condition) and (2) a second group of participants who were not warned of the presence of the sequence (incidental condition). The main purpose of this experiment was to confirm that explicit knowledge of the sequence may play a significant role in the SRT task. More specifically, our aims were to replicate previous results showing an increase in the difference between the learned and the random sequences when participants have an explicit knowledge of the sequence (e.g., Curran \& Keele, 1993) and to demonstrate that explicit knowledge of the sequence actually constitutes relevant information, which participants can use to improve their reaction times.

A generation task was administered after the SRT task in order to assess the explicit knowledge acquired by 
the participants during the learning session. The generation task we used differed from that of Destrebecqz and Cleeremans (2001), who asked their participants to freely generate a sequence of 96 items. Indeed, with such a procedure, it is difficult to interpret the parts of the generated sequence that are not correct; by definition, an incorrect response (given either by chance or, in the exclusion condition, because the participant recollected the target response and, therefore, gave another response) cannot be associated with the preceding one to serve as a cue for the next response, which will probably also be incorrect. How, then, should one interpret such responses? Another reason why we changed the procedure for the generation task is that, in Destrebecqz and Cleeremans's (2001) task, participants did not necessarily have to use an effortful strategy to remember whether they had already seen the sequence or not; for example, in the exclusion condition, one cannot rule out the possibility that some participants who were actually not able to consciously remember the sequence forced themselves to do anything other than what they were prompted to do by motor fluency (the generation-recognition bias described by RichardsonKlavehn, Gardiner, \& Java, 1996). In our task, we introduced a noise sequence in the generation task in order to obtain a real measure of the chance level in both the inclusion and the exclusion conditions; thus, we were able to determine whether or not the participants used different strategies in the inclusion and exclusion conditions. A difference between the baseline rates (chance level) in the inclusion and exclusion tests can be taken as direct evidence that the assumptions underlying the estimation procedure have been violated (see Jacoby, 1998). This differed from Destrebecqz and Cleeremans's (2001) study, in which the chance level was the calculated probability, which was, of course, equivalent for both conditions. ${ }^{2}$ Finally, another problem with a freely generated sequence is that participants can generate their sequences by using only some parts of the learned sequence; with such a procedure, it could be difficult to determine whether the participants had gained some knowledge of the whole sequence or only of parts of it.

For these reasons, we developed a new generation task based on the completion of fragments of sequences. In addition to the presentation of parts of the learned sequence, we added parts of a new sequence to the generation task, which allowed us to control for the difference in strategies in the inclusion and exclusion conditions. Another advantage of this procedure is that, when participants do not recognize a sequence in the generation task, they may believe it is a new one that they can complete with the first response that comes to mind. So, we expected that this task, which is more effortful than the free generation task, would be more sensitive to the dissociation between implicit and explicit processes.

As for the PDP, we expected, in the incidental condition, to replicate the results of the RSI conditions obtained by Destrebecqz and Cleeremans (2001), Destrebecqz (2004), and Wilkinson and Shanks (2004). Specifically, we expected to find the same pattern of results regard- ing the rate of completion in the inclusion and exclusion conditions (inclusion higher than exclusion and exclusion higher than chance).

\section{Method}

\section{Participants}

Forty undergraduate students from the University of Liège, 18-25 years of age, participated in the experiment; they were randomly assigned to one of the two learning conditions (with or without preliminary explicit learning of the sequence).

\section{Materials}

The experiment was run on a personal computer. Four white dots on a black background were constantly presented on a horizontal line. The dots were $6 \mathrm{~cm}$ apart from each other. The stimulus consisted of a white circle $1 \mathrm{~cm}$ in diameter that appeared $2 \mathrm{~cm}$ below one of the four dots.

\section{Procedure}

Preliminary explicit-learning phase. The participants in the explicit-learning condition first had to learn the sequence explicitly. Each of the eight elements of the sequence was presented consecutively on a computer screen at its particular location (among the four possible ones). The participants had to press the corresponding key of the keyboard, and then the next stimulus was displayed. The keys (C-V-B-N on an AZERTY keyboard) were spatially compatible with the locations of the stimuli on the screen. Once the sequence was completed, the participants had to repeat it. If the participants made an error, the computer made a noise, and the procedure began anew. The knowledge criterion was reached when the participants could repeat the sequence twice without mistakes. In order to ensure that the participants not only could recall the whole sequence, but also could "manipulate" their sequence knowledge, they also had to perfectly recall the following parts of the sequence separately: Locations $1-2-3-4,3-4-5-6,5-6-7-8$, and 7-8-1-2. So, both this selective recall and the recall of the whole sequence ensured that the participants had gained a full explicit knowledge of the sequence.

SRT task. After this explicit-learning phase, the SRT task began. The experiment consisted of 15 blocks of a four-choice RT task. Each block included 56 trials, for a total of 840 trials. On each trial, a stimulus appeared at one of the four possible locations, and the participants were asked to respond as quickly as possible to each stimulus by pressing the corresponding key of the keyboard. The participants had to respond with the middle and index fingers of the left hand for $\mathrm{C}$ and $\mathrm{V}$ and of the right hand for $\mathrm{B}$ and $\mathrm{N}$. The target was removed only when the correct key was pressed; so, if the participants made an error, they had to correct it. In this case, the RT encoded in the analysis was the RT for the correct response. The next stimulus appeared after a $250-\mathrm{msec}$ interval. The participants were given a break after each experimental block.

The participants were presented with one of the following two ambiguous sequences (in an ambiguous sequence, each position could be followed by two possibilities; i.e., in our first sequence, "D" could be followed by either "B" or "C"; A. Cohen, Ivry, \& Keele, 1990). Each sequence consisted of eight stimuli: "D B A C B D C A" and "A C D B C A B D." One experimental block consisted of eight repetitions of the sequence. In each condition, half of the participants were trained with the first sequence for the first 12 blocks and for Blocks 14 and 15 and with the second sequence for the 13th block; this design was reversed for the other half of the participants. Learning of the sequence during Blocks 1-12 would be attested by the longer RTs during Block 13. The task began with a series of 50 randomly generated practice trials.

After the SRT task, the participants were presented with 40 chunks of stimuli in both the inclusion and the exclusion conditions. Half of these chunks were fragments of the sequence that they had learned 
during the SRT task; the other half were fragments of a sequence that they had not seen before (lure sequence). The length of these chunks was from two to six elements. "D A B C B A D C" was the lure sequence for the Ambiguous 1 group, and "B A B C D A D C" for the Ambiguous 2 group. We used these lure sequences in order to avoid any interference effect due to the presentation of the other sequence during Block 13.

We tried to keep the PDP task as close as possible to the SRT task. The chunks were presented on the computer screen. The participants had to press the corresponding key of the keyboard for each trial. When the participants had to remember the two following trials, the arrows were presented alone. The participants could take all the time they wanted to respond in each trial.

In the inclusion condition, the participants had to complete the chunk with the next two elements of the learning sequence; in the exclusion condition, they had to complete the chunk with anything other than the next two elements of the learning sequence. If they could not remember which elements of the sequence followed the presented chunk, they had to complete it with the first two elements that came to mind. The advantage of completing the chunk with two elements (instead of one) is that, by reducing the computed chance level (.11 instead of .33), it increased the statistical power of our design (see McClelland, 1997). In the exclusion condition, the participants were explicitly asked to use no other strategy than remembering the sequence so they could avoid reproducing it. Instructions were repeated in the middle of each condition. Half the participants began with the inclusion condition, and the other half with the exclusion condition. Finally, for the generation task, the stimuli were arranged in two different orders. In each condition, one of the two orders of presentation was randomly assigned to the participants, so that no order effect could explain the results.

\section{Results}

\section{Reaction Time Task}

The RTs for the participants trained with Sequences 1 and 2 are combined in the following analyses. For 1 participant, we observed a slowing down of the RTs between Block 1 and Block 12 greater than two standard deviations from the mean; therefore, we removed his data from the analyses.

Figure 1 shows the median RTs for each block plotted separately for each learning condition.

An ANOVA with block (two levels: Block 12 vs. Block 13) as a within-participants variable and learning condition (two levels) as a between-participants variable showed a significant effect of the block variable $\left[F(1,37)=56.721, M S_{\mathrm{e}}=4,241, p<.001\right]$, indicating that the participants reacted more quickly in Block 12 than in Block 13; there was no significant learning condition effect $\left[F(1,37)=2.790, M S_{\mathrm{e}}=20,686, p=.103\right]$, but there was a significant interaction $[F(1,37)=6.652$, $\left.M S_{\mathrm{e}}=4,241, p<.05\right]$. This interaction seems to indicate that the difference between the learning sequence and the transfer sequence was greater for the participants in the explicit-learning condition than for the participants in the incidental-learning condition. To be sure that participants in the incidental-learning condition actually learned something about the sequence, we performed a planned comparison between Blocks 12 and 13 only for these participants. This analysis revealed a significant block effect $\left[F(1,37)=11.955, M S_{\mathrm{e}}=4,240.73, p<.01\right]$, which confirms that these participants showed sequence-specific learning as well.

However, one might argue that the knowledge acquired concerned only first-order transitions. Indeed, Sequences 1 and 2 differed on half of their transitions (i.e., A was followed by $\mathrm{C}$ and $\mathrm{D}$ in Sequence 1 and by $\mathrm{B}$ and $\mathrm{C}$ in Sequence 2). In this context, learning that A was followed by D might be sufficient to explain, at least in part, the participants' performance on both the SRT and the PDP tasks (mainly in the incidental-learning condition). In order to test the nature of the knowledge acquired, we compared the RTs for fragments that were common to the two sequences with the RTs for fragments that were not (see Table 1). If the participants acquired only first-order conditional information, they should react equally quickly for the common fragments in Blocks 12 and 13.

We performed an ANOVA with learning condition as a between-participants variable and with block (two levels: Block 12 or 13) and type of fragment (two levels: common or distinct) as within-participants variables. This analysis revealed no learning condition effect $[F(1,38)=1.724$, $M S_{\mathrm{e}}=41,700, p>1$ ], a significant effect for block $\left[F(1,38)=52.87, M S_{\mathrm{e}}=9,717, p<.001\right]$, confirming

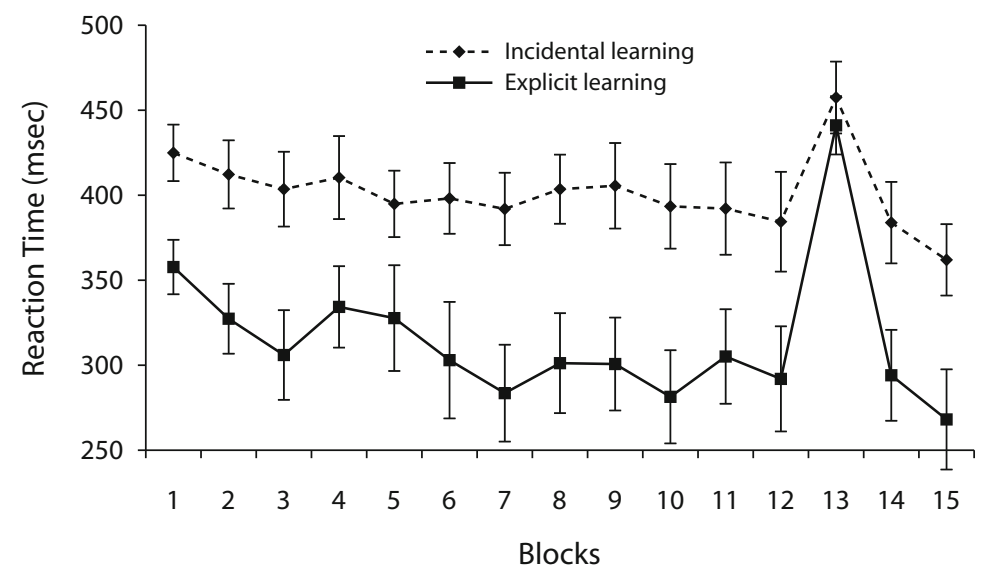

Figure 1. Mean reaction times (with $S E M$ s) for each block, plotted separately for the incidental-learning and explicit-learning conditions. 
Table 1

Mean Reaction Times (in Milliseconds; With Standard Errors of the Means) for the Common and Distinct Fragments in Blocks 12 and 13 for Both the Incidental- and the Explicit-Learning Conditions

\begin{tabular}{clccccc}
\hline \multirow{2}{*}{ Group } & Type of & \multicolumn{2}{c}{ Block 12 } & & \multicolumn{2}{c}{ Block 13 } \\
\cline { 3 - 4 } \cline { 6 - 7 } Explicit & Fragment & $M$ & SEM & & $M$ & SEM \\
\cline { 7 - 8 } Incidental & Common & 277.60 & 29.77 & & 433.85 & 22.36 \\
& Distinct & 333.12 & 29.75 & & 495.50 & 22.50 \\
& Common & 368.92 & 28.45 & & 413.17 & 18.44 \\
& Distinct & 418.55 & 30.75 & & 509.00 & 23.15 \\
\hline
\end{tabular}

that RTs are shorter for Block 12 than for Block 13, and a significant effect for type of fragment $[F(1,38)=56.57$, $\left.M S_{\mathrm{e}}=3,048, p<.001\right]$, showing that common fragments were processed more quickly than distinct fragments. There was also a significant block $\times$ learning condition interaction $\left[F(1,38)=56.57, M S_{\mathrm{e}}=9,717, p=.005\right]$, indicating that the difference between Block 12 and Block 13 was greater for the participants in the explicit-learning condition than for the participants in the incidental-learning condition; a significant block $\times$ type of fragment interaction $\left[F(1,38)=6.18, M S_{\mathrm{e}}=1,107, p=.01\right]$, showing that the type of fragment effect was greater in Block 13 than in Block 12; and no type of fragment $\times$ learning condition interaction $\left[F(1,38)=0.655, M S_{\mathrm{e}}=3,048, p>.2\right]$. Regarding the block $\times$ type of fragment interaction, a planned comparison revealed that common fragments were processed more quickly in Block 12 than in Block 13 $\left[F(1,38)=37.44, M S_{\mathrm{e}}=5,367.2, p<.001\right]$. Finally, the analysis showed a marginally significant block $X$ type of fragment $\times$ learning condition interaction $\left[F(1,38)=6.18, M S_{\mathrm{e}}=1,107, p=.064\right]$, indicating that the block $\times$ type of fragment effect was not equivalent between learning conditions. Thus, it is possible that the difference between Block 12 and Block 13 for the common fragments was due only to the explicit-learning participants. Planned comparisons confirmed that common fragments were processed more quickly in Block 12 than in Block 13, not only in the explicit-learning condition $\left[F(1,38)=45.48, M S_{\mathrm{e}}=5,367.2, p<.001\right]$, but also in the incidental-learning condition $\left[F(1,38)=7.11, M S_{\mathrm{e}}=\right.$ $4,741.07, p<.05]$. This result is important because, if the difference between Block 12 and Block 13 for the common fragments was due to the participants in the explicitlearning condition alone, it could be the result of their earlier explicit learning of the sequence, rather than of the acquisition of ambiguous information. The fact that this difference was also observed for participants in the incidental-learning condition confirms that they learned higher order conditional information.

This analysis means that, even if the participants did learn first-order transitions, the information acquired also reflects, at least partly, the learning of ambiguous associations, which were more complex than first-order transitions (see also A. Cohen et al., 1990, who suggested that the processes involved in the learning of ambiguous relations differ from those involved in the acquisition of first-order relations).

\section{PDP Task}

In the generation task, one point was given when the two elements generated by the participant corresponded to the learned sequence (i.e., a correct response in the inclusion condition and an error in the exclusion condition).

Table 2 shows the mean proportions of completion under inclusion instructions and exclusion instructions and the chance level.

With regard to the chance levels, we first examined whether the level of completion of the lure chunks was the same with inclusion and exclusion instructions. We performed an ANOVA with instruction (two levels: inclusion vs. exclusion) as a within-participants variable and learning condition (two levels: incidental vs. explicit learning) as a between-participants variable. This analysis revealed no significant effect (all $p \mathrm{~s}>.05$ ), which indicates (1) that the chance level was equivalent under both the inclusion and the exclusion instructions and, therefore, that it would not be necessary to correct the estimation of implicit processes by subtracting the chance level (because of this equivalence, the data for the chance level will be combined for the following analyses), and (2) that both groups used the same response criterion for responding under the inclusion and exclusion instructions. The analysis on the instruction conditions yielded a medium effect size (.56); in order to reach a power level of .80, a sample size of 78 would be needed. This seems to indicate that the participants used the same strategy under the different conditions and with different instructions.

Note that one could claim that some differences between the lure sequence and the learning sequence could have led us to understate the chance level; indeed, the lure sequence contained some salient chunks (such as A-B-C) that were not present in the training sequence. We agree that this possibility cannot be totally ruled out and that, if we had underestimated the chance levels, our interpretation of the PDP results would have been unsuitable. However, this does not seem to have been the case. Indeed, previous SRT studies

Table 2

Proportions (Means and Standard Errors of the Means) of Chunks Completed With Their Corresponding Elements in the Learned Sequence for the Two Learning Groups (Incidental and Explicit) in the Two Instruction Conditions (Inclusion and Exclusion) and for the Chance Levels (C.L.)

\begin{tabular}{|c|c|c|c|c|c|c|c|c|}
\hline \multirow[b]{2}{*}{ Group } & \multicolumn{2}{|c|}{ Inclusion } & \multicolumn{2}{|c|}{ Exclusion } & \multicolumn{2}{|c|}{ C.L. Incl. } & \multicolumn{2}{|c|}{ C.L. Excl. } \\
\hline & $M$ & SEM & $M$ & SEM & $M$ & $\overline{S E M}$ & $M$ & $\overline{S E M}$ \\
\hline Incidental learning & .186 & .030 & .147 & .018 & .081 & .020 & .155 & .024 \\
\hline Explicit learning & .425 & .060 & .150 & .025 & .127 & .022 & .145 & .023 \\
\hline
\end{tabular}


in which the PDP has been applied have used the calculated chance level (and not the observed chance level, as in the present study) as a baseline. In the present experiment, the completion level of the lure sequence (.127) was above the calculated chance level (which was .11), confirming that the chance levels were not underestimated. Nevertheless, we agree that, because of the structural differences between the learning sequence and the lure sequence, the interpretation of the results should be taken with caution.

Then we performed an ANOVA with instructions (two levels: inclusion vs. exclusion) as a within-participants variable and learning condition (two levels: incidental learning vs. explicit learning) as a between-participants variable, in order to compare the level of completion for the old chunks. This analysis revealed a significant effect for instructions $\left[F(1,37)=14.76, M S_{\mathrm{e}}=0.033, p<.001\right]$, a significant effect of learning condition $\left[F(1,37)=12.88, M S_{\mathrm{e}}=0.022\right.$, $p<.001]$, and a significant interaction $[F(1,37)=8.27$, $\left.M S_{\mathrm{e}}=0.033, p<.01\right]$. In other words, the number of correct completions was higher under the inclusion than under the exclusion instructions; the participants in the explicitlearning condition completed the sequences correctly more often (independently of the instructions), and the decrease in correct completions for the exclusion completion was larger for the participants in the explicit-learning condition than for those in the incidental-learning condition.

To determine whether performance is due to implicit or explicit processes, Destrebecqz and Cleeremans (2001) focused on two aspects: the difference between the chance level and the level of completions under exclusion instructions and the decrease in correct completions between the inclusion and the exclusion instruction conditions. So, following Destrebecqz and Cleeremans (2001), we first compared the levels of completion under inclusion and exclusion instructions with the observed chance level. For the incidental-learning condition, we performed a one-tailed $t$ test in order to determine whether the performance with the inclusion instructions was different from the chance level; this analysis revealed a significant difference $[t(18)=2.476, p<.05]$. We then compared the exclusion condition with the chance level; in this case, the difference was not significant $[t(18)=1.273, p>.10]$.

The same comparisons were performed for the explicitlearning condition. These analyses showed a significant difference between inclusion and the chance level $[t(19)=$ $4.610, p<.001$ ], and no difference between the exclusion condition and the chance level $[t(19)=0.596, p=.279]$.

Then we performed planned comparisons between the inclusion and exclusion instructions for each learning condition in the ANOVA described above. These analyses revealed that the decline in performance between inclusion and exclusion instructions was not significant in the incidental-learning condition $\left[F(1,37)=0.45, M S_{\mathrm{e}}=\right.$ $0.033, p>.50]$, whereas it was in the explicit-learning condition $\left[F(1,37)=23.17, M S_{\mathrm{e}}=0.033, p<.001\right]$. Note that this absence of a significant decrease in the incidental-learning condition contrasts with Destrebecqz and Cleeremans's (2001) results, which showed a significant decrease between the inclusion and exclusion conditions and no difference between the chance level and the exclusion condition in their RSI condition. This difference might be related to the number of trials used in the SRT task: 840 learning trials in our study and approximately 1,400 in Destrebecqz and Cleeremans's (2001) study.

\section{Discussion}

As was expected, the participants in the explicit-learning condition, who were trained to gain a full explicit knowledge of the sequence, responded more quickly in the SRT task than did participants in the incidental-learning condition. These results, which suggest that explicit knowledge of the sequence constitutes relevant knowledge that participants can use to perform efficiently in an SRT task, confirm the results obtained in previous studies (Curran \& Keele, 1993; Doyon, Owen, Petrides, Sziklas, \& Evans, 1996) that showed shorter RTs in a deterministic SRT task for participants who had some explicit knowledge of the sequence. These results also provide additional evidence that the SRT paradigm cannot be considered as a purely implicit learning task.

Nevertheless, one could argue from these results that although explicit mechanisms undeniably seem to play a role in sequence learning, this does not mean that implicit mechanisms are not involved as well. It may be the case, depending on the constraints of the task, that the relative contributions of implicit and explicit mechanisms can vary. In the present case, the type of instruction given to the participants may have determined the balance between implicit and explicit mechanisms. This point of view is consistent with Cleeremans's (1997) proposal that conscious and unconscious cognitive processes are described better in terms of a continuum than from an all-or-nothing perspective.

The PDP offers one way to explore the relative contributions of explicit and implicit knowledge to participants' performance. With the PDP, one could predict from our results that participants in the explicit-learning condition should perform better in the inclusion condition than with the participants in the incidental-learning condition. Moreover, if performance on the SRT task is sustained mainly by explicit processes, we should observe that the participants in the explicit learning condition are better at avoiding producing parts of the sequence under exclusion instructions than are the participants in the incidentallearning condition. On the whole, if explicit processes play the greatest role in participants' performance, these processes should be stronger in the explicit-learning than in the incidental-learning condition.

The first prediction was confirmed: The participants' performance in response to inclusion instructions was far better in the explicit-learning condition than in the incidental-learning condition.

On the other hand, the second prediction was not confirmed: Performance levels in the exclusion condition were not better for the participants in the explicit-learning condition. If one believes that performance in this task is sustained mainly by explicit processes, this observation is surprising. If the participants in the explicit-learning 
condition had a better explicit knowledge of the sequence, why were they not more able than the participants in the incidental-learning condition to avoid producing parts of the sequence in the generation task?

One possibility is that, following Wilkinson and Shanks's (2004) point of view, performance on the generation task is not sustained by implicit processes. According to these authors, the exclusion condition is more difficult than the inclusion condition, and this could explain why the participants performed at chance levels in the exclusion condition. (They would respond in the exclusion condition by giving the first location that came to mind, instead of trying to avoid producing the sequence, in which case their performance would not differ from the chance level because, according to this point of view, they would have no implicit sequence knowledge.) Note that, with such an interpretation, one should also consider that the participants absolutely did not try to avoid producing the sequence (if they had tried and succeeded on at least some trials, their performance should have tended to be below the chance level), which seems quite unlikely.

The other possibility is that both explicit and implicit processes were involved in the participants' performance during the generation task. In the exclusion condition, for some items that they were able to consciously remember, they avoided producing the sequence. For other items, either because they could not remember them or because of the difficulty of the exclusion instructions, they responded randomly, and so they completed the sequence on the basis of automatic processes. If this was the case, possibly their correct responses based on their explicit sequence knowledge (i.e., responses not corresponding to the sequence) were counterbalanced by their incorrect responses based on automatic processes (i.e., responses corresponding to the sequence). And the result of these "contradictory" responses is a performance level similar to the chance level (for the participants in both the incidental- and the explicit-learning conditions).

\section{EXPERIMENT 2}

In the first experiment, we showed that explicit knowledge of the sequence improves performance on an SRT task. It could be suggested that this improvement is possible because a deterministic sequence allows the use of explicit strategies. One hypothesis is that when the sequence can be maintained in working memory, participants are able to use this knowledge to respond more rapidly to the stimulus, probably because they can anticipate their response to the next stimulus. But the basic idea underlying this study is that, even if it is proved that explicit mechanisms may play a role in SRT performance, this does not mean that the SRT paradigm is an explicit task per se. Our hypothesis is that participants' ability to use explicit strategies in the SRT task depends on the characteristics of the task, including the RSI, the kind of instructions given to participants, or the disruption of the sequence by the interpolation of random elements between each presentation of the deterministic sequence (e.g., Meulemans et al., 1998).
The purpose of our second experiment was to explore again the effect of the preliminary instructions given to the participants (preliminary explicit learning of the sequence vs. incidental learning), but this time with a probabilistic sequence, instead of a deterministic one. Our prediction was that even though the participants would be able to learn the characteristics of the sequence in long-term memory, they would not be able to make use of this knowledge in the SRT task, because of the probabilistic nature of the sequence. With a probabilistic sequence, unlike a deterministic one, the amount of relevant information that has to be maintained in working memory is probably too large for participants to be able to use it online during the SRT task (and to make their anticipation judgments quickly). So a probabilistic sequence should prevent the use of explicit strategies in the SRT task. More specifically, if only implicit processes can be used in this task, we expect that the effect of learning, as measured by RTs, will be the same under both learning conditions.

The probabilistic sequence we used in this experiment was inspired by Schvaneveldt and Gomez (1998). To ensure that the participants acquired a full knowledge of the sequence, half of the ones who belonged to the explicitlearning group learned both the probable and the improbable transitions (see Schvaneveldt \& Gomez, 1998); the other half of the explicit-learning group learned only the probable transitions and was informed that the sequence would contain irregularities. This was done in order to prevent knowledge of the irregularities from interfering with their response strategy; it is also possible that the full knowledge of both probable and improbable transitions would exceed the participants' processing capacities. Finally, this condition is also interesting because it was closer to that in Experiment 1, in which we showed that this preliminary sequence knowledge is relevant for the SRT performance (in both experiments, these participants learned the same sequence, without irregularities).

\section{Method}

\section{Participants}

Sixty undergraduate students, 18-26 years of age, were randomly assigned to one of the three experimental conditions: incidental learning, probable-sequence learning (explicit P group), probableand improbable-sequence learning (explicit PI group).

\section{Materials}

The materials were the same as those in Experiment 1.

\section{Procedure}

The procedure was rather similar to the one used in Experiment 1, except that the sequence was probabilistic, instead of deterministic. The probabilistic sequence used in this experiment was characterized by a ratio of probable/improbable elements of $80 \%$ to $20 \%$ for half the sequence locations (this means that only half of the locations in the sequence might be followed by an improbable location); thus, the total proportion of improbable trials was $10 \%$. This procedure was motivated by the fact that the participants had to learn the sequence and its irregularities explicitly; if improbable elements could have followed each of the sequence elements, it would have been too difficult for them to learn this information explicitly. Thus, this choice is explained by the need to facilitate explicit learning as much as possible. Note that, when an improbable location occurred, the 
Table 3

Two Ambiguous Sequences Used in Experiment 2 and Their Irregularities

\begin{tabular}{|c|c|c|c|c|c|}
\hline \multicolumn{3}{|c|}{$\begin{array}{c}\text { Sequence } 1 \\
\text { (D B A C B D C A) }\end{array}$} & \multicolumn{3}{|c|}{$\begin{array}{c}\text { Sequence } 2 \\
\text { (A C D B C A B D) }\end{array}$} \\
\hline & $80 \%$ & $20 \%$ & & $80 \%$ & $20 \%$ \\
\hline DBA & $\mathrm{C}$ & $\mathrm{D}$ & $\mathrm{ACD}$ & B & $\mathrm{A}$ \\
\hline $\mathrm{ACB}$ & $\mathrm{D}$ & A & $\mathrm{DBC}$ & A & D \\
\hline BDC & A & B & $\mathrm{CAB}$ & D & $\mathrm{C}$ \\
\hline CAD & B & $\mathrm{C}$ & BDA & $\mathrm{C}$ & $\mathrm{B}$ \\
\hline
\end{tabular}

Note-The irregular element located at Positions 4, 6, 8, and 2 is shown in the " $20 \%$ " column.

next element was not the one that simply followed it in the series but was always generated consistently with the new context (see Table 3 for the sequences and their irregularities). This was also explained to the participants during the preliminary explicit-learning phase. The Appendix gives an example of how an irregular item was created.

Before the beginning of the task, the participants in the P condition and participants in the PI condition were asked to learn the sequence. The same knowledge criterion as that in Experiment 1 was used for both groups. In the P condition, the participants were actually informed that the sequence would contain irregularities during the task. In the PI condition, the participants were also shown the irregularities in the sequence and were told how they were created. In addition to the knowledge criterion mentioned above, these participants had to successfully recall the irregular parts of the sequence once. Specifically, they were asked to recall Locations $1-2-3-4$, Locations 3-4-5-6, Locations 5-6-7-8, and Locations 7-8-1-2 when Locations 4, 6, 8, and 2, respectively, were irregular.

The task was composed of 15 blocks of 60 trials, for a total of 900 trials. During Blocks 1-12, 14, and 15, half the participants had to respond to the stimuli (probable and improbable) belonging to Sequence 1. Block 13 was composed of stimuli (probable and improbable) corresponding to Sequence 2 (transfer sequence). For the remaining participants, Sequence 2 was the learning sequence, and Sequence 1 the transfer sequence.

The PDP task was exactly the same as in Experiment 1.

\section{Results}

\section{Reaction Time Task}

We first analyzed our data by comparing RTs for probable and improbable elements of the sequence. The data from 1 participant in the explicit PI learning group were removed because they were more than three standard deviations from the mean difference between the probable and the improbable locations (calculated for his group separately). Table 4 shows the median RTs for each type of item (regular vs. irregular) described separately for each of the three learning conditions. We performed an ANOVA with type of item (two levels: regular items vs. irregular items) as a within-participants variable and learning condition (three levels: incidental, explicit $\mathrm{P}$, or explicit PI) as a between-participants variable. This analysis revealed that neither type of item $[F(1,56)=1.394$, $\left.M S_{\mathrm{e}}=464, p=.242\right]$ nor learning condition $[F(2,56)=$ $\left.1.538, M S_{\mathrm{e}}=4,114, p=.223\right]$ was significant. On the other hand, the interaction was significant $[F(2,52)=$ $\left.3.892, M S_{\mathrm{e}}=464, p=.02\right]$. This interaction shows that the relations between regular and irregular trials were different for the different learning groups. The incidentallearning and explicit P learning groups processed the irregular trials more quickly than the regular ones, whereas the PI explicit learning group processed the regular trials quicker. One might see a learning effect in the pattern of results observed in the explicit PI learning condition. To determine whether this learning effect was significant, we performed a planned comparison with type of item (two levels) as a within-participants condition for only the explicit PI learning condition. This analysis revealed that the effect was not significant $\left[F(1,57)=2.04, M S_{\mathrm{e}}=\right.$ 463.765, $p=.157]$.

Because no difference was observed for the regular or irregular items in the different learning groups, we examined another learning index: the difference between Block 12 (the last training block) and Block 13 (the transfer block).

Figure 2 shows the median RTs for each block plotted separately for each of the three learning conditions.

We performed an ANOVA with Block (Block 12 vs. Block 13) as a within-participants variable and learning condition (three levels: incidental, explicit P, or explicit PI) as a between-participants variable. This analysis revealed a significant block effect $[F(2,57)=120.375$, $\left.M S_{\mathrm{e}}=882, p<.001\right]$, a marginal effect for learning condition $\left[F(1,57)=2.552, M S_{\mathrm{e}}=6,628, p=.086\right]$, and no interaction $\left[F(2,57)=0.287, M S_{\mathrm{e}}=882, p=.752\right]$. Because learning condition was marginally significant (and because the participants in the incidental-learning condition were a bit slower than the participants in the explicit PI learning condition), we performed a planned comparison to ensure that no difference existed between the incidental-learning condition and the explicit PI learning condition. This analysis revealed that there was no difference $\left[F(1,57)=2.30, M S_{\mathrm{e}}=6,628, p>.10\right]$.

These results indicate that the participants learned the sequence, as is shown by the increase between Block 12 and Block 13; moreover, and contrary to Experiment 1, this learning effect was not improved by preliminary explicit knowledge of the sequence. This is confirmed by the absence of a learning condition effect and by the planned comparison between the incidental-learning condition and the explicit PI condition. Interestingly, it appears that, on the whole, the slowest participants were those in the $\mathrm{P}$ (probable-sequence learning) condition; although not statistically significant, this observation contradicts the idea that explicit preliminary knowledge of the sequence must improve RTs in an SRT task.

However, another possibility is that this absence of an effect was related to a lack of statistical power. This could be particularly true for the participants in the explicit P learning condition, for whom the learning effect (i.e.,

Table 4

Mean Reaction Times (in Milliseconds; With Standard Errors of the Means) for the Regular and Irregular Locations in the Three Learning Conditions

\begin{tabular}{|c|c|c|c|c|}
\hline \multirow[b]{2}{*}{ Group } & \multicolumn{2}{|c|}{ Regular } & \multicolumn{2}{|c|}{ Irregular } \\
\hline & $M$ & SEM & $M$ & SEM \\
\hline Incidental learning & 379.5 & 11.52 & 362.6 & 9.80 \\
\hline Explicit P learning & 393.4 & 11.52 & 386.3 & 9.80 \\
\hline Explicit PI learning & 360.7 & 11.82 & 370.7 & 10.05 \\
\hline
\end{tabular}

Note-P, probable sequence learning; PI, probable/improbable sequence learning. 


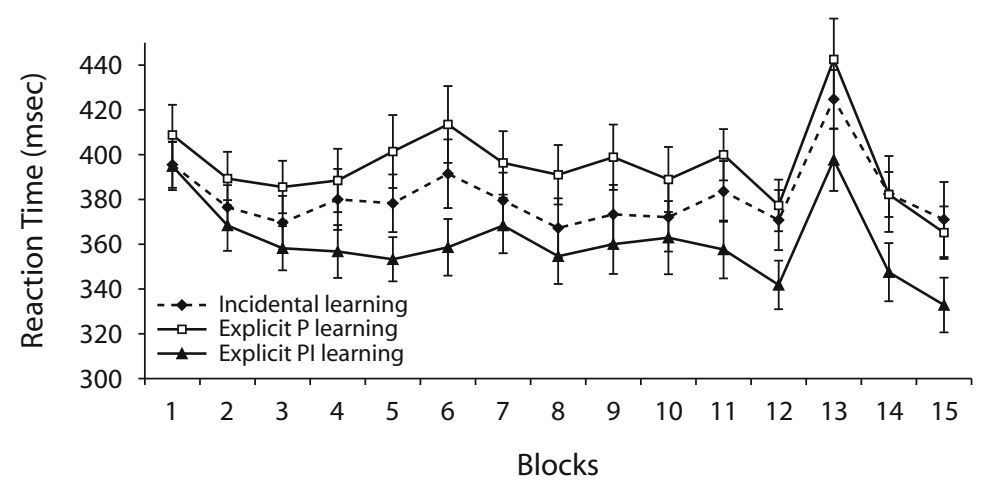

Figure 2. Mean reaction times (with $S E M$ s) for each block, plotted separately for the incidental-learning, probable-sequence learning (P), and probable/ improbable sequence learning (PI) conditions.

the difference between Blocks 12 and 13) was greater than that for the participants in the incidental learning condition (contrary to the participants in the explicit PI learning condition, for whom the learning effect was smaller than that in the incidental-learning condition). Following J. Cohen (1988), the effect size observed between the explicit $\mathrm{P}$ learning condition and the incidental-learning condition (.1278) can be considered small. To obtain a significant difference between the groups, each group would have had to contain 293 participants. So it seems that our results are not related to a lack of power and are in accordance with the results of previous studies in which participants who had to learn the sequence before performing a probabilistic SRT task did not show any significant learning effect (e.g., Cleeremans \& Jiménez, 1998).

As in Experiment 1, we tested whether the participants learned only first-order transitions. If this were the case, it could explain the absence of an effect of learning group. In other words, if performance can be improved by exploiting only first-order dependencies, which are simple pieces of information, the absence of any effect of learning condition may turn out to be trivial (if the sequence is too easy to learn, RTs in both the explicit and the incidental conditions could rapidly reach their maximum; this floor effect would lead to an absence of difference between the learning groups).

Again as in Experiment 1, we compared the RTs for fragments that were common to the two sequences with the RTs for fragments that were not (see Table 5). If the participants acquired only first-order conditional information, they should react as quickly for the common fragments in Block 12 as for those in Block 13. To test this hypothesis, we performed an ANOVA with learning condition (three levels: incidental, explicit P, or explicit PI) as a between-participants variable and block (two levels: Block 12 or Block 13) and type of fragment (two levels: common or distinct) as within-participants variables. This analysis revealed a marginally significant learning condition effect $\left[F(1,57)=3.107, M S_{\mathrm{e}}=13,820, p=\right.$ $.052]$, indicating a tendency for the participants in the explicit PI learning condition to process the trials more quickly than did the participants in the explicit P learn- ing condition; a significant effect for block $[F(1,57)=$ $\left.126.363, M S_{\mathrm{e}}=1,621, p<.001\right]$, indicating that RTs were shorter for Block 12 than Block 13; and a significant type of fragment effect $\left[F(1,57)=5.802, M S_{\mathrm{e}}=\right.$ $1,099, p<.05]$, showing that common fragments were processed more quickly than distinct fragments. There was no learning condition $\times$ block interaction $[F(2,57)=$ $\left.0.686, M S_{\mathrm{e}}=1,621, p>.1\right]$; a significant learning condition $\times$ type of fragment interaction $[F(2,57)=7.476$, $\left.M S_{\mathrm{e}}=1,099, p<.005\right]$, showing that the difference between the type of fragments was observed mainly in the explicit $\mathrm{P}$ group; and no block $\times$ type of fragment interaction $\left[F(1,57)=1.791, M S_{\mathrm{e}}=1,079, p>.1\right]$, suggesting that the RT difference between Blocks 12 and 13 was the same for both types of fragments. Finally, there was a significant learning condition $\times$ block $\times$ type of fragment interaction $\left[F(2,57)=3.33, M S_{\mathrm{e}}=1,079, p=\right.$ $.042]$. Regarding the latter interaction, the point was to determine whether the difference between Block 12 and Block 13 for common fragments would be observed in all the learning conditions (and not merely for the participants in the explicit-learning conditions). Planned comparisons performed on the data for the participants in both the explicit P and PI learning conditions and in the incidental-learning condition confirmed that their RTs were shorter for common fragments in Block 12 than in Block $13\left[F(1,57)=39.31, M S_{\mathrm{e}}=1,218.03, p<.001\right.$,

Table 5

Mean Reaction Times (in Milliseconds; With Standard Errors of the Means) for the Common and Distinct Fragments in Blocks 12 and 13 for the Incidental-Learning and the Two Explicit-Learning Conditions

\begin{tabular}{clccccc}
\hline \multirow{2}{*}{ Group } & Type of & \multicolumn{2}{c}{ Block 12 } & & \multicolumn{2}{c}{ Block 13} \\
\cline { 3 - 4 } \cline { 6 - 7 } Fragments & $M$ & SEM & & $M$ & SEM \\
\hline Incidental & Common & 383.95 & 12.15 & & 424.65 & 14.96 \\
& Distinct & 367.80 & 15.18 & & 438.35 & 12.18 \\
Explicit P & Common & 366.20 & 12.21 & & 435.40 & 15.79 \\
& Distinct & 404.05 & 12.21 & & 462.80 & 16.21 \\
Explicit PI & Common & 344.60 & 12.21 & & 392.95 & 15.79 \\
& Distinct & 348.25 & 14.81 & & 399.80 & 16.21 \\
\hline
\end{tabular}

Note-P, probable sequence learning; PI, probable/improbable sequence learning. 
and $F(1,57)=19.19, M S_{\mathrm{e}}=1,218.03, p<.001$, for the $\mathrm{P}$ and PI learning conditions, respectively; $F(1,57)=13.59$, $M S_{\mathrm{e}}=1,218.03, p<.001$, for the incidental-learning condition], which indicates that the participants actually learned ambiguous transitions. Moreover, the fact that the RTs for the common fragments were shorter than the RTs for the distinct fragments within Block 13 suggests that first-order transitions were learned as well.

\section{PDP Task}

As in Experiment 1, we used the PDP to examine whether the participants' knowledge of the sequence at the end of the task could be considered implicit or explicit.

We first compared the chance levels obtained in the different conditions (i.e., the proportions of completions corresponding to the new sequence for the new chunks). The aim of this analysis was to determine whether the participants used different strategies under the inclusion and the exclusion instructions. If the difference between inclusion and exclusion is significant, it is possible that participants do not give the first response that comes to mind when they do not recognize the sequence. We performed an ANOVA with instructions (two levels: inclusion vs. exclusion) as a within-participants variable, and learning condition (3 levels: incidental, explicit $\mathrm{P}$, or explicit PI) as a between-participants variable. This analysis showed no significant difference for the instruction variable $\left[F(1,57)=0.003, M S_{\mathrm{e}}=0.009, p>.90\right]$ but a significant effect for learning condition $[F(2,57)=$ 4.313, $\left.M S_{\mathrm{e}}=0.011, p<.05\right]$ and a marginally significant interaction $\left[F(2,57)=2.877, M S_{\mathrm{e}}=0.009, p=\right.$ $.065]$. Note that the analysis for the instruction conditions yielded a small effect size (.17). Planned comparisons reveal that the chance level differed significantly between the incidental and explicit $\mathrm{P}$ conditions $[F(1,57)=4.908, p<.05]$ and between the incidental and explicit PI conditions $[F(1,57)=7.715, p<.01]$, but there was no difference between the two explicitlearning conditions $[F(1,57)=0.316, p>.50]$. Thus, these results seem to indicate that the participants did not use different strategies under the instruction conditions. It is possible that the difference observed between the experimental conditions was related to differences in the attentional investment between groups. In a related context, Mulligan (2003) showed that attention plays a role in the perceptual-priming effect. If one considers that the first fragments of the lure sequence might play the role of a visual prime for the subsequent fragments, it is possible that the priming effect for the last fragments was different between conditions because of differences in the attentional investment between the groups. More specifically, the participants in the explicit-learning groups may have been more attentive to the generation task because they adopted a more effortful strategy in order to distinguish between the new and the old chunks. Since the participants in the incidental condition were less conscious of the presence of a sequence during the SRT task, they relied more on their intuition, which was less effortful. In any case, the important point is the absence of any difference between the inclusion and exclusion conditions; consequently, these results have been combined for further analyses.

Table 6 shows the mean proportions of completion with inclusion instructions and exclusion instructions and the chance level. This table is particularly interesting because it shows the very low levels of completion with the exclusion instructions, which are below the chance level for both the explicit P and the explicit PI learning conditions. This means that participants who have an explicit knowledge of the sequence are able to avoid producing it. The results in Experiment 2 show clearly (and more clearly than in Experiment 1) that the knowledge acquired in both explicit-learning conditions is mainly explicit. Although the knowledge acquired in Experiment 1 was mainly explicit as well, it is nevertheless possible that the participants in Experiment 1 acquired more implicit knowledge than did the participants in Experiment 2, which could explain why they were unable to respond below the chance level in the exclusion condition.

We then performed an ANOVA with instructions (two levels: inclusion vs. exclusion) as a within-participants variable and learning condition (three levels: incidental learning, explicit P learning, or explicit PI learning) as a between-participants variable. This analysis revealed a significant effect for instructions $[F(1,57)=98.26$, $\left.M S_{\mathrm{e}}=0.037, p<.001\right]$, a significant effect for learning condition $\left[F(2,57)=22.46, M S_{\mathrm{e}}=0.019, p<.001\right]$, and a significant interaction $\left[F(1,57)=23.66, M S_{\mathrm{e}}=0.037\right.$,

Table 6

Proportions (Means and Standard Errors of the Means) of Chunks Completed With Their Corresponding Elements in the Learned Sequence for the Three Learning Groups (Incidental, Explicit P, and Explicit PI) in the Two Instruction Conditions (Inclusion and Exclusion) and for the Chance Levels (C.L.)

\begin{tabular}{|c|c|c|c|c|c|c|c|c|}
\hline \multirow[b]{2}{*}{ Group } & \multicolumn{2}{|c|}{ Inclusion } & \multicolumn{2}{|c|}{ Exclusion } & \multicolumn{2}{|c|}{ C.L. Incl. } & \multicolumn{2}{|c|}{ C.L. Excl. } \\
\hline & $M$ & SEM & $M$ & SEM & $M$ & SEM & $M$ & $S E M$ \\
\hline Incidental & .172 & .021 & .156 & .016 & .131 & .028 & .114 & .016 \\
\hline Explicit P & .534 & .066 & .090 & .021 & .146 & .024 & .205 & .023 \\
\hline Explicit PI & .656 & .051 & .074 & .017 & .208 & .023 & .170 & .019 \\
\hline
\end{tabular}

Note-In the explicit P learning condition, the participants had only to learn explicitly the more probable transitions of the sequence; in the explicit PI learning condition, they had to learn explicitly both the probable and the improbable transitions of the sequence (see the text for details). 
$p<.001]$. The completion rate was shown to be higher for the inclusion than for the exclusion instructions. Planned comparisons revealed that the participants in the explicit $\mathrm{P}$ and explicit PI learning conditions completed the sequence correctly more often (independently of the instructions) than did those in the incidental-learning condition $\left[F(1,57)=22.76, M S_{\mathrm{e}}=0.019, p<.001\right.$, and $F(1,57)=41.76, M S_{\mathrm{e}}=0.019, p<.001$, respectively] but that there was no difference between the explicit $\mathrm{P}$ learning condition and the explicit PI learning condition $\left[F(1,57)=2.86, M S_{\mathrm{e}}=0.019, p=.096\right]$.

To determine the nature of the knowledge acquired during the task, we first compared the inclusion scores with the chance level for each of the three conditions. For all three conditions, the one-tailed $t$ test was significant $[t(19)=1.771, p<.05 ; t(19)=5.17, p<.001 ; t(19)=$ $8.558, p<.001$, for the incidental, explicit $\mathrm{P}$, and explicit PI learning conditions, respectively]. Thus, this analysis reveals that for each of the conditions, the participants learned something about the sequence.

Then we compared the exclusion scores with the chance level for each of the conditions. For both of the explicitlearning conditions, the exclusion scores were significantly lower than the chance level $[t(19)=2.77, p=.01$, and $t(19)=6.01, p<.001$, for the explicit $\mathrm{P}$ and explicit PI conditions, respectively]. For the incidental condition, the difference was not significant $[t(19)=1.49, p>.10]$.

To see whether the exclusion scores were significantly lower than the inclusion scores, we performed planned comparisons between the inclusion and exclusion instructions for each learning condition in the ANOVA described above. These analyses revealed that performance in the incidental-learning condition did not decrease significantly between the inclusion and exclusion conditions $[F(1,57)=$ $\left.0.069, M S_{\mathrm{e}}=0.036, p>.75\right]$, contrary to the performance of the participants in the two explicit conditions, whose scores decreased significantly between the inclusion and exclusion conditions [for the $\mathrm{P}$ condition, $F(1,57)=$ 53.43, $M S_{\mathrm{e}}=0.036, p<.001$, and for the PI condition, $\left.F(1,57)=92.08, M S_{\mathrm{e}}=0.036, p<.001\right]$. This decrease had the same magnitude for both explicit-learning conditions $\left[F(1,57)=2.61, M S_{\mathrm{e}}=0.037, p>.10\right]$.

These results suggest that in the generation task, the performance of the participants in the explicit $\mathrm{P}$ and explicit PI conditions was sustained mainly by explicit knowledge of the sequence. For the incidental condition, the results suggest that the participants had less sequence knowledge than did those in the explicit conditions. The nature of the knowledge acquired by the participants in the incidental condition is less clear. It is possible that their performance in the generation task was sustained by both implicit and explicit knowledge. Indeed, for the participants in the explicit-learning conditions, the explicit knowledge of the sequence led to exclusion levels below the chance levels. Thus, for the participants who performed above the chance level in the inclusion condition and who were unable to achieve exclusion completion below the chance level, one can conclude that their knowledge was, at least partly, implicit. Another interpretation could be that since the exclu- sion rates did not differ from the baseline, the participants acquired only a little explicit knowledge. Our inability to reach a clear conclusion on the nature of the knowledge acquired by the participants in the incidental condition may have been due to certain limitations of the PDP itself.

\section{Discussion}

The purpose of Experiment 2 was to examine whether explicit knowledge of the sequence could improve performance on a probabilistic SRT task. Our hypothesis was that this explicit preliminary learning would have no impact on RTs, because a probabilistic sequence prevents the use of explicit strategies during the SRT task.

In Experiment 1, we showed that the explicit knowledge of the sequence provided relevant information to perform efficiently in the SRT task. In Experiment 2, because there was no RT difference between the probable and the improbable locations, one conclusion could be that the participants did not actually learn the sequence. However, this conclusion is not supported by the comparison of the RTs for Blocks 12 and 13 (the transfer block), which showed an effect of the block variable (indicating that RTs improved from one block to the other) but no effect of learning condition and no interaction. These results suggest that the participants learned the sequence, whatever condition they belonged to (with or without preliminary explicit learning); they also suggest that the participants who explicitly learned the sequence before the SRT task were unable to use their explicit knowledge to improve their performance, even if this preliminary explicit knowledge included full awareness of the probabilistic characteristics of the sequence (i.e., both its probable and its improbable transitions). Consequently, because explicit knowledge does not help participants to perform better on the SRT task, the only way to explain the decrease in their RTs is to call on implicit-learning mechanisms.

One might argue that the presence of a sequence, instead of random trials, in the transfer block (Block 13) could contribute to an explanation of the absence of a difference between the conditions; indeed, it could be that the participants in the explicit conditions, because their attention was directed to the detection and learning of regularities, showed greater learning in Block 13 than did the other participants, which might explain the pattern of results in Experiment 2. However, if this hypothesis were true, we should have observed the same phenomenon in Experiment 1 (in which the learning effect for the transfer sequence should even be more pronounced, because of the deterministic nature of the sequence), which was not the case: In Experiment 1, we obtained the same performance levels in the transfer block for the participants in the explicit- and incidental-learning conditions.

We must still try to understand why we did not observe any difference between probable and improbable locations, contrary to what is usually shown in studies using a probabilistic SRT paradigm. One possible explanation is linked to the number of trials involved in the task. When we compared our Experiment 2 with other studies (e.g., Jiménez et al., 1996; Rowland \& Shanks, 2006; Schvane- 
veldt \& Gomez, 1998), we found that those studies contained at least twice the number of trials we used in Experiment 2. Although further studies will be necessary to understand more precisely why we did not observe any difference between probable and improbable locations, it remains the case that, to some extent, specific sequences actually occurred, as is shown by the other learning index: the difference between the learning block and the transfer block (see, e.g., Soetens, Melis, \& Notebaert, 2004), showing that the participants processed trials in Block 12 more quickly than in Block 13.

It could also be argued that Shanks and St. John's (1994) information criterion was not respected. In this case, the information given to the participants may have been irrelevant. However, one might think that if this information was relevant in Experiment 1, it should be relevant in Experiment 2 as well. Nevertheless, it remains possible that the participants in the probabilistic condition could not use their knowledge of the sequence because they were not shown the whole sequence during the SRT task but saw only chunks of the sequence. In this case, the relevant information should be chunks of sequence, rather the whole sequence. However, in our experiment, the participants had to learn not only the sequence as a whole, but also all the possible chunks of the sequence; thus, the information criterion would still be met.

Another piece of information that could be relevant for performing the task is simply that the participants in the explicit conditions were informed of the presence of a sequence (even though Cleeremans \& Jiménez, 1998, showed that participants could not use such information in a probabilistic task).

Whatever the case may be, the fact that a particular piece of knowledge is relevant does not mean that it can be used. We think that the point is that explicit use of this knowledge was less economical than using implicitlearning mechanisms, because, even if relevant, the explicit information was unusable for the participants in Experiment 2.

The results of the generation task show that the participants in the incidental-learning condition did not acquire much knowledge of the sequence. This was not the case for the participants in the explicit-learning condition, who obtained high inclusion scores and very low exclusion scores. Such results clearly confirm that these participants used their explicit knowledge of the sequence to perform the generation task.

Why did the participants in the explicit-learning conditions in Experiment 2 reveal the intervention of explicit processes during the generation task more clearly than did the participants in the explicit-learning condition in Experiment 1 ? One possible explanation is that participants in the explicit-learning conditions in Experiment 2 gained a better knowledge of the sequence than did the participants in the explicit-learning condition in Experiment 1. If this was the case (which would be shown by high inclusion scores), it would be impossible to explain a belowchance exclusion score without calling on the intervention of significant explicit processes. To test this possibility, we compared the inclusion scores of the participants in the explicit-learning condition in Experiment 1 with those of the participants in the explicit $P$ learning condition in Experiment 2 (these two conditions were exactly the same in terms of the preliminary explicit learning of the sequence, whereas the participants in the explicit PI learning condition also had to learn the irregularities of the sequence). However, the result of the $t$ test was not significant, and so this first hypothesis was not confirmed $[t(38)=1.22$, $p=.225]$.

Another explanation is that the amount of knowledge acquired by the participants in both experiments was equivalent but the nature of this knowledge differed between experiments. In other words, in Experiment 1, the absence of irregularities may have favored the automation of the preliminary explicit knowledge acquired more than in Experiment 2 . This automation could explain why the participants in Experiment 1 found it more difficult to avoid producing the sequence under exclusion instructions.

Another point that we would like to emphasize is related to the validity of the PDP itself for assessment of the knowledge acquired during the SRT task. Indeed, the results of Experiment 2 revealed no difference between learning conditions with regard to performance during the SRT task, a result that we may interpret as indicating that the participants in the explicit-learning conditions could not use their explicit knowledge of the sequence to improve their RTs during the SRT task. However, despite this lack of SRT difference between groups, there was a difference in the PDP-based task. In all probability, this difference can be attributed only to the initial explicit sequence learning; in this case, what the PDP measured was this initial knowledge, and not the knowledge acquired during the SRT task. These considerations are not insignificant: What we show is that an explicit task, even if it is sensitive to some aspects of participants' knowledge, can be of no help in the assessment of the knowledge acquired during the SRT task itself. We believe that this point is not relevant only for situations in which participants learn the sequence explicitly before performing the SRT task. It is another way of expressing the more general problem of the explicit posttask assessment of the knowledge acquired during implicit-learning tasks: Our results suggest that the explicit knowledge elicited with such tasks can sometimes be considered a by-product of the (implicit) learning task itself, and not at all as a determinant of performance on the implicit-learning task.

\section{GENERAL DISCUSSION}

Our study goes to the heart of the controversy regarding the possibility that sequence knowledge acquisition occurs outside of consciousness. To explore this question, we manipulated the information given to participants before they performed the SRT task: Some of them performed the task without any preliminary knowledge of the sequence (the classical incidental-learning situation), whereas others were asked to learn the sequence explicitly beforehand. The results of the two experiments 
reported here confirm that preliminary explicit knowledge improves RTs when the sequence is deterministic but has no impact on sequence learning when the sequence is probabilistic.

Jiménez et al. (1996) obtained similar results, but, in the present study, participants were able to gain full knowledge of the sequence, despite its irregularities in the probabilistic condition. In our study, the participants did not have any time limit to learn the sequence, and they had to fulfill the knowledge criterion. These points are central because the aim of the study was to match Shanks and St. John's (1994) information criterion as closely as possible.

Another important aspect of this study was that it characterized the knowledge acquired in a probabilistic SRT task better by using Jacoby's (1991) PDP. Applied to a subsequent cued generation task, the PDP suggests that when participants are not informed of the presence of regularities in the sequence, the improvement in their RTs is, at least, based less on explicit mechanisms than when they are informed, and maybe partly sustained, by implicit-learning processes.

Our study is also supported by the findings of Rauch et al. (1995), who showed that the neuronal correlates of explicit and implicit learning were different. In this context, we might hypothesize that the primary visual and inferior parietal cortices (which are specific to explicit learning) would not have been involved in the participants' performance in Experiment 2, neither in the incidental-learning nor in the explicit-learning condition; indeed, as we have shown, the participants in the explicit-learning conditions could not use the explicitly learned information. On the other hand, according to Rauch et al.'s findings, the right ventral premotor cortex, right ventral caudate, right thalamus, and bilateral visual association cortex, activated during implicit learning, could have been activated in Experiment 2. We might also hypothesize that in Experiment 1, regions implied in both explicit and implicit learning were activated for the explicit-learning group, and maybe for the incidental-learning group as well.

This study provides new evidence for the debate regarding the nature-implicit versus explicit — of the processes involved in SRT tasks. It shows that explicit processes may play a major role in the acquisition of a determinist sequence, although learning is mainly implicit when the sequence is probabilistic.

According to Shanks and St. John (1994), the hypothesis that sequence learning might occur outside of consciousness is not supported by the evidence. In line with this position is the observation of a correlation between certain measures of explicit knowledge and the RT improvement in the so-called "implicit" sequence-learning task (e.g., a correlation between the level of explicit knowledge in an explicit recognition task and the RT improvement in the SRT task). Other data reported in the literature reinforce this interpretation: Some studies have shown that participants who are informed of the presence of a sequence perform better on the SRT task than do participants who are not given such preliminary information.
We believe that our results run counter to this point of view and that this study supports the idea that implicit and explicit mechanisms are dissociated in sequence learning. Indeed, we have designed a situation in which, although they possessed the relevant explicit information to carry out the SRT task, participants were not able to actually use it to improve their performance; more specifically, in our second experiment, the preliminary explicit knowledge of the sequence (and of all of its probable and improbable transitions) did not confer any advantage in the SRT task, in comparison with the SRT performance of participants who were not informed of the presence of regularities in the sequence. So the decrease in RTs during the SRT task in both conditions (with and without preliminary explicit knowledge) cannot be explained by the intervention of explicit mechanisms; on the contrary, we argue that it can be explained better by the acquisition of knowledge outside of consciousness. Note that one could consider that the absence of above-chance exclusion scores for participants in the incidental group contradicts this affirmation (see Wilkinson \& Shanks, 2004), and that the generation task and the SRT task could simply be differentially sensitive to explicit sequence knowledge. However, this view is not shared by many authors dealing with the PDP. Indeed, in different studies, the authors have reported exclusion scores below chance levels and, nevertheless, have concluded that automatic processes played a role in the performance (e.g., Bergerbest \& Goshen-Gottstein, 2002; Jacoby, Toth, \& Yonelinas, 1993).

On the whole, the results of our study give rise to different conclusions, some of which are (apparently) contradictory. First, our results confirm the results of previous studies showing the important role that explicit mechanisms can play in participants' performance on SRT tasks. The results of our Experiment 1 undoubtedly confirm that, in an SRT task, RTs may be significantly improved when participants have the opportunity to explicitly learn the sequence before performing the task, indicating that such explicit knowledge can be considered relevant for performing such tasks efficiently. Although it would be interesting to determine the importance of such an influence in other conditions (e.g., when the RSIs are reduced to zero), this study provides new evidence that, at least in some cases, explicit mechanisms may play an important role in SRT performance.

Second, this study also suggests that, in some conditions, implicit mechanisms also play a crucial role in the task; one of these conditions is the statistical structure of the sequence: In Experiment 2, we showed that a probabilistic sequence (unlike a deterministic one) cannot benefit from explicit knowledge of the transitions that characterize the sequence. And finally, this study sheds new light on the results obtained with the explicit posttask measures that are usually administered after implicit-learning tasks. Indeed, although we showed that the participants in the explicit conditions who performed a probabilistic SRT task had a high level of explicit knowledge - which was confirmed in the generation task by their high level of 
completion in the inclusion condition and the low level of completion in the exclusion condition - the learning curve of these participants did not differ from the curve obtained by the participants in the implicit learning condition. So, a measure sensitive to the explicit knowledge possessed by the participants can be of no help in determining the knowledge acquired during the SRT task, which permitted the improvement in RTs (and more specifically, a better improvement for the repeating sequence than for the transfer sequence). This indicates that, in implicitlearning tasks, the fact that explicit knowledge can be demonstrated with an explicit posttask measure does not necessarily mean that participants used this knowledge to perform the "implicit" learning task. It also suggests that a positive correlation between this explicit knowledge and task performance cannot be viewed a priori as a proof that performance was determined by the explicit knowledge. As we have already suggested, the reverse is also possible: Development of explicit knowledge could be the result of efficient learning based on implicit-learning mechanisms. As was suggested by Destrebecqz and Cleeremans (2003), depending on the different parameters of the task, the knowledge acquired might be the result of a continuous mechanism characterized by gradual improvements in the quality of representations, which gradually become stronger and more available to conscious control.

\section{AUTHOR NOTE}

This research was supported by the National Fund for Scientific Research (Belgium). We are grateful to Pierre Perruchet, Arnaud Destrebecqz, and Benjamin Clegg for their fulfilling comments on the previous versions of the manuscript. Correspondence concerning this article should be addressed to N. Stefaniak, Department of Cognitive Sciences, Boulevard du Rectorat 3, 4000 Liège, Belgium (e-mail: nicolas .stefaniak@ulg.ac.be).

\section{REFERENCES}

Bergerbest, D., \& Goshen-Gottstein, Y. (2002). The origins of levels-of-processing effects in a conceptual test: Evidence for automatic influences of memory from the process-dissociation procedure. Memory \& Cognition, 30, 1252-1262.

Cherry, K. E., \& Stadler, M. A. (1995). Implicit learning of a nonverbal sequence in younger and older adults. Psychology \& Aging, 10, 379-394.

Cleeremans, A. (1997). Principles for implicit learning. In D. C. Berry (Ed.), How implicit is implicit learning? (pp. 195-234). Oxford: Oxford University Press.

Cleeremans, A., \& Jiménez, L. (1998). Implicit sequence learning: The truth is in the details. In M. A. Stadler \& P. A. Frensch (Eds.), Handbook of implicit learning (pp. 323-364). Thousand Oaks, CA: Sage.

Cohen, A., Ivry, R. I., \& Keele, S. W. (1990). Attention and structure in sequence learning. Journal of Experimental Psychology: Learning, Memory, \& Cognition, 16, 17-30.

CoHen, J. (1988). Statistical power analysis for the behavioral sciences (2nd ed.). Hillsdale, NJ: Erlbaum.

Curran, T. (1997a). Effects of aging on implicit sequence learning: Accounting for sequence structure and explicit knowledge. Psychological Research, 60, 24-41.

Curran, T. (1997b). Higher-order associative learning in amnesia: Evidence from the serial reaction time task. Journal of Cognitive Neuroscience, 9, 522-533.

Curran, T., \& Hintzman, D. G. (1995). Violations of the independence assumption in process dissociation. Journal of Experimental Psychology: Learning, Memory, \& Cognition, 21, 531-547.

Curran, T., \& Keele, S. W. (1993). Attentional and nonattentional forms of sequence learning. Journal of Experimental Psychology: Learning, Memory, \& Cognition, 19, 189-202.

DESTREBECQZ, A. (2004). The effect of explicit knowledge on sequence learning: A graded account. Psychologica Belgica, 44, 217-247.

Destrebecqz, A., \& Cleeremans, A. (2001). Can sequence learning be implicit? New evidence with the process dissociation procedure. Psychonomic Bulletin \& Review, 8, 343-350.

Destrebecqz, A., \& Cleeremans, A. (2003). Temporal factors in sequence learning. In L. Jiménez (Ed.), Attention and implicit learning (pp. 181-213). Amsterdam: Benjamins.

Doyon, J., Owen, A. M., Petrides, M., Sziklas, V., \& Evans, A. C. (1996). Functional anatomy of visuomotor skill learning in human subjects examined with positron emission tomography. European Journal of Neuroscience, 8, 637-648.

Frensch, P. A., \& Miner, C. S. (1994). Effects of presentation rate and individual differences in short-term memory capacity on an indirect measure of serial learning. Memory \& Cognition, 22, 95-110.

Grafton, S. T., Hazeltine, E., \& Ivry, R. (1995). Functional mapping of sequence learning in normal humans. Journal of Cognitive Neuroscience, 7, 497-510.

Hartman, M., Knopman, D. S., \& Nissen, M. J. (1989). Implicit learning of new verbal associations. Journal of Experimental Psychology: Learning, Memory, \& Cognition, 15, 1070-1082.

Hintzman, D. L., \& CurRan, T. (1997). More than one way to violate independence: Reply to Jacoby and Shrout (1997). Journal of Experimental Psychology: Learning, Memory, \& Cognition, 23, 511-513.

Howard, D. V., \& Howard, J. H., JR. (2001). When it does hurt to try: Adult age differences in the effects of instructions in implicit pattern learning. Psychonomic Bulletin \& Review, 8, 798-805.

JACOBY, L. L. (1991). A process dissociation framework: Separating automatic from intentional uses of memory. Journal of Memory \& Language, 30, 513-541.

JACOBY, L. L. (1998). Invariance in automatic influences of memory: Toward a user's guide for the process-dissociation procedure. Journal of Experimental Psychology: Learning, Memory, \& Cognition, 24, 3-26.

JACOBY, L. L., \& Shrout, P. E. (1997). Toward a psychometric analysis of violations of the independence assumption in process dissociation. Journal of Experimental Psychology: Learning, Memory, \& Cognition, 23, 505-510.

Jacoby, L. L., Toth, J. P., \& Yonelinas, A. P. (1993). Separating conscious and unconscious influences of memory: Measuring recollection. Journal of Experimental Psychology: General, 122, 139-154.

Jiménez, L., MéndeZ, C., \& Cleeremans, A. (1996). Comparing direct and indirect measures of sequence learning. Journal of Experimental Psychology: Learning, Memory, \& Cognition, 22, 948-969.

Keele, S. W., Ivry, R., Mayr, U., Hazeltine, E., \& Heuer, H. (2003). The cognitive and neural architecture of sequence representation. Psychological Review, 110, 316-339.

McClelland, G. H. (1997). Optimal design in psychological research. Psychological Methods, 2, 3-19.

Meulemans, T., Van der Linden, M., \& Perruchet, P. (1998). Implicit sequence learning in children. Journal of Experimental Child Psychology, 69, 199-221.

Mulligan, N. W. (2003). Effects of cross-modal and intramodal division of attention on perceptual implicit memory. Journal of Experimental Psychology: Learning, Memory, \& Cognition, 29, 262-276.

Nissen, M. J., \& Bullemer, P. (1987). Attentional requirements of learning: Evidence from performance measures. Cognitive Psychology, 19, 1-32.

Nissen, M. J., Willingham, D., \& Hartman, M. (1989). Explicit and implicit remembering: When is learning preserved in amnesia? Neuropsychologia, 27, 341-352.

Perruchet, P., Bigand, E., \& Benoit-Gonin, F. (1997). The emergence of explicit knowledge during the early phase of learning in sequential reaction time tasks. Psychological Research, 60, 4-13.

Perruchet, P., Chambaron, S., \& Ferrel-Chapus, C. (2003). Learning from implicit learning literature: Comment on Shea, Wulf, Whitacre, and Park (2001). Quarterly Journal of Experimental Psychology, 56A, 769-778.

Rauch, S. L., Savage, C. R., Brow, H. D., Curran, T., Alpert, N. M., Kendrick, A., ET AL. (1995). A PET investigation of implicit and explicit sequence learning. Human Brain Mapping, 3, 271-286. 
Reber, P. J., \& Squire, L. R. (1994). Parallel brain systems for learning with and without awareness. Learning \& Memory, 1, 217-229.

Reber, P. J., \& SQuire, L. R. (1998). Encapsulation of implicit and explicit memory in sequence learning. Journal of Cognitive Neuroscience, 10, 248-263.

Richardson-Klavehn, A., Gardiner, J. M., \& Java, R. I. (1996). Memory: Task dissociations, process dissociations, and dissociations of consciousness. In G. Underwood (Ed.), Implicit cognition (pp. 85158). Oxford: Oxford University Press.

Rowland, L., \& SHanKs, D. R. (2006). Sequence learning and selection difficulty. Journal of Experimental Psychology: Human Perception \& Performance, 32, 287-299.

SchVAneveldT, R. W., \& Gomez, R. L. (1998). Attention and probabilistic sequence learning. Psychological Research, 61, 175-190.

SHANKs, D. R. (2005). Implicit learning. In K. Lamberts \& R. Goldstone (Eds.), Handbook of cognition (pp. 202-220). London: Sage.

Shanks, D. R., \& ST. John, M. F. (1994). Characteristics of dissociable human learning systems. Behavioral \& Brain Sciences, 17, 367-447.

Shea, C. H., Wulf, G., Whitacre, C. A., \& Park, J. H. (2001). Surfing the implicit wave. Quarterly Journal of Experimental Psychology, 54A, 841-862.

Soetens, E., Melis, A., \& Notebaert, W. (2004). Sequence learning and sequential effects. Psychological Research, 69, 124-137.

Stadler, M. A., \& Frensch, P. A. (1994). Whither learning, whither memory? Behavioral \& Brain Sciences, 17, 423-424.

Stadler, M. A., \& Roediger, H. L., III (1998). The question of awareness in research on implicit learning. In M. A. Stadler \& P. A. Frensch (Eds.), Handbook of implicit learning (pp. 105-132). Thousand Oaks, CA: Sage.
Thomas, K. M., \& Nelson, C. A. (2001). Serial reaction time learning in preschool- and school-age children. Journal of Experimental Child Psychology, 79, 364-387.

Wilkinson, L., \& Shanks, D. R. (2004). Intentional control and implicit sequence learning. Journal of Experimental Psychology: Learning, Memory, \& Cognition, 30, 354-369.

Willingham, D. B., \& Goedert-Eschmann, K. (1999). The relation between implicit and explicit learning: Evidence for parallel development. Psychological Science, 10, 531-534.

Willingham, D. B., Greeley, T., \& Bardone, A. M. (1993). Dissociation in a serial response time task using a recognition measure: Comment on Perruchet and Amorim (1992). Journal of Experimental Psychology: Learning, Memory, \& Cognition, 19, 1424-1430.

Willingham, D. B., Nissen, M. J., \& Bullemer, P. (1989). On the development of procedural knowledge. Journal of Experimental Psychology: Learning, Memory, \& Cognition, 15, 1047-1060.

\section{NOTES}

1. It must be pointed out that some aspects of the procedure are controversial (see, e.g., Curran \& Hintzman, 1995; Hintzman \& Curran, 1997; and for a reply, see Jacoby, 1998; Jacoby \& Shrout, 1997). However, it is not possible to discuss these debates in detail here, since this is not the principal purpose of this article.

2. For example, a statistically lower chance level in the exclusion condition than in the inclusion condition could reflect the fact that the participants adopted a more prudent strategy in the exclusion condition in order to avoid producing an error. The response criterion is, therefore, different in the two conditions, and in such a case, results of the PDP could not be interpreted.

\section{APPENDIX}

The two sequences are "D B A C B D C A" and "A C D B C A B D"

An improbable location in the fourth position of the first sequence (this position corresponds to the location "C," which follows the locations "D B A") will consistently be the location " $\mathrm{D}$," because "D" is the location that follows the other " $\mathrm{A}$ " in the sequence. So, we took into consideration the position that precedes the location " $\mathrm{C}$ " (i.e., "A") and chose the item that follows this same location in the sequence (i.e., "D," because the other "A" of the sequence is followed by "D"). Now, the first sequence begins with "D B A D," instead of "D B A C." After this irregular item, the sequence continues as the normal sequence (i.e., the irregular location " $\mathrm{D}$ " is followed by "B" because, in the sequence, the locations "A D" are followed by "B"). In this context, the complete sequence with an irregular location on the fourth location is "D B A D B A C B D C A."

(Manuscript received May 24, 2007;

revision accepted for publication May 19, 2008.) 